\title{
Joint Source and Relay Precoding Designs for MIMO Two-Way Relay Systems
}

\author{
Rui Wang and Meixia Tao \\ Department of Electronic Engineering \\ Shanghai Jiao Tong University, Shanghai, 200240, P. R. China \\ Emails: \{liouxingrui, mxtao\}@sjtu.edu.cn
}

\begin{abstract}
We investigate the source and relay precoding based on the minimum mean-square-error (MSE) criterion for amplifyand-forward (AF) MIMO two-way relay systems. This joint design problem is a highly nonconvex optimization problem. In this work, we present two efficient design algorithms. The first one aims to minimize the total MSE of the two users by alternatively solving three sub-problems and it is iterative in nature. The second design holds a specific precoder structure, which is able to parallelize channels in both the Multiple Access (MAC) and Broadcast (BC) phases of two-way relaying. Based on such structure, the joint precoding design is simply reduced to the joint source and relay power allocation problem. The efficiency of both proposed algorithms is verified via simulation.
\end{abstract}

\section{INTRODUCTION}

Relay based transmission has been considered as a promising technique to offer significant benefits including throughput enhancement, coverage extension and power reduction in wireless communications. The recently proposed two-way relaying is shown able to further improve the usage of channel resources by applying the idea of network coding in the physical layer [1]. In contrast to the conventional one-way relaying, where four time slots are needed for information exchange between two source nodes due to the half-duplex constraint, the two-way relay procotol can use only two times slots. On the other hand, the multi-input-multi-output (MIMO) technique is known capable of enhancing the system capacity linearly by providing extra spatial dimensions. Therefore, incorporating the MIMO technique into the two-way relay system is expected to further increase the system throughput.

For the MIMO two-way relay system, various forwarding strategies at the relay have been studied. The relay can completely decode the received bit sequence pair, combine them using, for example, XOR or superposition coding, and then broadcast them to the two destinations in a regenerative manner. This is known as decode-and-forward (DF) relay strategy. In [2], the authors investigate and compare the capacity gain for two different re-encoding operations in DF based MIMO two-way systems. In [3], the boundary of capacity region of Gaussian MIMO two-way relay broadcast channels is derived. Another type of processing at the relay is non-regenerative, referred to as amplify-and-forward (AF).

This work is supported by the NSF of China under grant 60902019, the Joint Research Fund for Overseas Chinese, Hong Kong and Macao Young Scholars under grant 61028001, and the Innovation Program of Shanghai Municipal Education Commission under grant 11ZZ19.
Compared to the DF relay strategy, the AF relay strategy is attractive for its simplicity. In [4], the authors develop one algorithm to compute the globally optimal beamforming matrix at the relay node and characterize the system capacity region. In [5], applying the gradient descent algorithm, the authors propose an iterative scheme to find the suboptimal relay precoder with the aim of maximizing the sum-rate of AF MIMO two-way relay systems. In [6], the authors propose a transceive precoding scheme at the relay node by using zero-forcing $(\mathrm{ZF})$ and minimum mean-square-error (MMSE) criteria with certain antenna configurations.

In this study, we focus on the joint linear precoding design at both source and relay nodes for MIMO two-way relaying with AF strategy. In contrast to [4], we consider a two-way relay system that both the source and relay nodes are equipped with multiple antennas. Furthermore, we study the joint source and relay precoding design rather than relay precoding design only. Although the joint precoding design for traditional oneway AF relay system has been well studied (see, e.g. [7], [8]), to our best knowledge, the work on the joint precoding design at both the source and relay nodes for two-way relay systems is very limited in the literature.

Our goal is to jointly design the precoders for source and relay nodes for minimizing the mean-square-error (MSE) at the destinations. Unfortunately, it is shown that the MMSEbased objective function is highly nonconvex with respect to the precoding matrices and thus the optimal solution may not be trackable in an efficient way. To overcome the difficulty, we first decouple the joint design problem into three sub-problems and then propose an iterative algorithm to alternatively solve the three sub-problems. Since the solution for each subproblem can be obtained optimally, the convergence is thus ensured. Furthermore, a lower-complexity channel parallelization based precoding design algorithm under certain antenna configurations is proposed. This method applies two joint matrix decomposition techniques and hence has a specific precoder structure. This structure is able to parallelize channels in both the Multiple Access (MAC) and Broadcast (BC) phases in the two-way relay system. Based on the corresponding precoder structures, the joint precoding design is simply reduced to the joint source and relay power allocation problem.

Notations: $\mathcal{E}[\cdot]$ denotes expectation over the random variables within the brackets. $\otimes$ denotes the Kronecker operator. $\operatorname{vec}(\cdot)$ and $\operatorname{mat}(\cdot)$ signify the matrix vectorization operator 


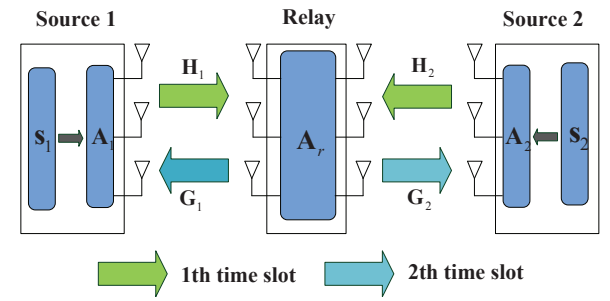

Fig. 1. Illustration of the MIMO two-way relay system.

and the corresponding inverse operation, respectively. $\operatorname{Tr}(\boldsymbol{A})$ and $\boldsymbol{A}^{-1}$ stand for the trace and the inverse of matrix $\boldsymbol{A}$, respectively. Superscripts $(\cdot)^{T}$ and $(\cdot)^{H}$ denote transpose and conjugate transpose. $\Re(z)$ denotes the real part of $z$. $\mathbb{C}^{x \times y}$ denotes the space of $x \times y$ matrices with complex entries. The distribution of a circular symmetric complex Gaussian vector with mean vector $\boldsymbol{x}$ and covariance matrix $\boldsymbol{\Sigma}$ is denoted by $\mathcal{C N}(\boldsymbol{x}, \boldsymbol{\Sigma})$.

\section{SySTEM MODEL}

It is assumed that two source nodes $S_{1}$ and $S_{2}$ want to exchange messages through the relay node $R$ within two time slots, as shown in Fig. 1. In the first time slot (also referred to as the MAC phase), the two source nodes $S_{1}$ and $S_{2}$ simultaneously transmit signals to the relay node at the same frequency band. After receiving the signals transmitted in the first time slot, the relay performs a linear processing by multiplying them with a precoding matrix and then forwards it in the second time slot (also referred to as the BC phase). We further assume that the source nodes are both equipped with $N$ antennas and the relay node is equipped with $N_{r}$ antennas. To fully use the multiplexing gain, it is supposed that $N$ datastreams are transmitted from each of the sources.

Based on the above assumptions, the transmitted signal vectors from the source nodes can be expressed as $\boldsymbol{x}_{i}=\boldsymbol{A}_{i} \boldsymbol{s}_{i}$, for $i=1,2$. Here, $\boldsymbol{s}_{i} \in \mathbb{C}^{N \times 1}$ and $\boldsymbol{A}_{i} \in \mathbb{C}^{N \times N}$ denote the modulated signal vector and the transmit precoding matrix, respectively, at source $S_{i}$. We further assume that the entries in $\boldsymbol{s}_{i}$ are normalized to have unit power, i.e., $\mathcal{E}\left(\boldsymbol{s}_{i} \boldsymbol{s}_{i}^{H}\right)=\boldsymbol{I}_{N}$. In addition, it is supposed that the maximum transmission power at $S_{1}$ and $S_{2}$ are $\tau_{1}$ and $\tau_{2}$, i.e.,

$$
\operatorname{Tr}\left(\boldsymbol{A}_{1} \boldsymbol{A}_{1}^{H}\right) \leq \tau_{1}, \quad \operatorname{Tr}\left(\boldsymbol{A}_{2} \boldsymbol{A}_{2}^{H}\right) \leq \tau_{2} .
$$

After the two-user MAC phase, the received $N_{r} \times 1$ signal vector at the relay is denoted as $\boldsymbol{y}_{r}=\boldsymbol{H}_{1} \boldsymbol{x}_{1}+\boldsymbol{H}_{2} \boldsymbol{x}_{2}+\boldsymbol{n}_{r}$ with $\boldsymbol{H}_{i} \in \mathbb{C}^{N_{r} \times N}$ being the MIMO channel matrix from $S_{i}$ to $R$, and $\boldsymbol{n}_{r} \sim \mathcal{C N}\left(\mathbf{0}, \sigma_{r}^{2} \boldsymbol{I}_{N_{r}}\right)$ representing the additive noise vector.

Upon receiving $\boldsymbol{y}_{r}$, the relay amplifies it by multiplying it with a precoding matrix $\boldsymbol{A}_{r} \in \mathbb{C}^{N_{r} \times N_{r}}$. Then, the $N_{r} \times 1$ transmitted signal vector at the relay node can be expressed as $\boldsymbol{x}_{r}=\boldsymbol{A}_{r} \boldsymbol{y}_{r}$. Here, we assume that the maximum transmission power at the relay node is $\tau_{r}$, i.e.,

$$
\operatorname{Tr}\left\{\boldsymbol{A}_{r}\left(\sum_{i=1}^{2} \boldsymbol{H}_{i} \boldsymbol{A}_{i} \boldsymbol{A}_{i}^{H} \boldsymbol{H}_{i}^{H}+\sigma_{r}^{2} \boldsymbol{I}_{N_{r}}\right) \boldsymbol{A}_{r}^{H}\right\} \leq \tau_{r},
$$

where we have used the assumption that the source signals and the relay noise are independent with each other. Then the relay broadcasts $\boldsymbol{x}_{r}$ to the destination nodes and the received signals at $S_{i}$ can be written as, after subtracting the self-interference

$$
\boldsymbol{y}_{i}=\boldsymbol{G}_{i} \boldsymbol{A}_{r} \boldsymbol{H}_{\bar{i}} \boldsymbol{A}_{\bar{i}} \boldsymbol{s}_{\bar{i}}+\boldsymbol{G}_{i} \boldsymbol{A}_{r} \boldsymbol{n}_{r}+\boldsymbol{n}_{i}, \quad i=1,2
$$

where $\bar{i}=2$ if $i=1$ and $\bar{i}=1$ if $i=2, \boldsymbol{G}_{i} \in \mathbb{C}^{N \times N_{r}}$ is the channel matrix from $R$ to $S_{i}$, and $\boldsymbol{n}_{i}$ denotes the additive noise vector at $S_{i}$ with $\boldsymbol{n}_{i} \sim \mathcal{C N}\left(\mathbf{0}, \sigma_{i}^{2} \boldsymbol{I}_{N}\right)$. Here, we have assumed that the global channel-state-information (CSI) and the designed precoding matrices are perfectly known at all the nodes and hence the back-propagating self-interference can be completely subtracted.

\section{Design Method 1: Iterative Precoding Design BASED ON MMSE CRITERION}

Based on the received signal vectors in (3) and assuming linear receiver at each destination node, the MSE expression at $S_{i}$ can be written as

$$
J_{i}=\mathcal{E}\left\{\left\|\boldsymbol{W}_{i} \boldsymbol{y}_{i}-s_{\bar{i}}\right\|_{2}^{2}\right\},
$$

where $\boldsymbol{W}_{i} \in \mathbb{C}^{N \times N}$ is the linear decode matrix at the destination. Substituting (3) into (4) further yields

$$
\begin{aligned}
J_{i}= & \operatorname{Tr}\left\{\boldsymbol{W}_{i} \boldsymbol{G}_{i} \boldsymbol{A}_{r} \boldsymbol{H}_{\bar{i}} \boldsymbol{A}_{\bar{i}} \boldsymbol{A}_{\bar{i}}^{H} \boldsymbol{H}_{\bar{i}}^{H} \boldsymbol{A}_{r}^{H} \boldsymbol{G}_{i}^{H} \boldsymbol{W}_{i}^{H}\right. \\
& -\boldsymbol{W}_{i} \boldsymbol{G}_{i} \boldsymbol{A}_{r} \boldsymbol{H}_{\bar{i}} \boldsymbol{A}_{\bar{i}}-\boldsymbol{A}_{\bar{i}}^{H} \boldsymbol{H}_{\bar{i}}^{H} \boldsymbol{A}_{r}^{H} \boldsymbol{G}_{i}^{H} \boldsymbol{W}_{i}^{H} \\
& \left.+\sigma_{r}^{2} \boldsymbol{W}_{i} \boldsymbol{G}_{i} \boldsymbol{A}_{r} \boldsymbol{A}_{r}^{H} \boldsymbol{G}_{i}^{H} \boldsymbol{W}_{i}^{H}+\sigma_{i}^{2} \boldsymbol{W}_{i} \boldsymbol{W}_{i}^{H}+\boldsymbol{I}_{N}\right\} .
\end{aligned}
$$

The problem is to find the optimal precoding/decoding matrices $\left\{\boldsymbol{A}_{r}, \boldsymbol{A}_{i}, \boldsymbol{W}_{i}, i=1,2\right\}$ such that the total MSE of the two users can be minimized. This is formulated as

$$
\min _{\boldsymbol{A}_{r}, \boldsymbol{A}_{i}, \boldsymbol{W}_{i}, i=1,2} J=J_{1}+J_{2} \text {, s.t. }
$$

Before solving (6), we present the following theorem.

Theorem 1 $1^{1}$ : To support $N$ data-streams in the considered system, the relay antenna number $N_{r}$ should at least be equal to $N$. Otherwise, for any power constraints at the sources and relay, the sum MSE $J_{1}+J_{2}$ is always larger than $2\left(N-N_{r}\right)$.

We next try to solve the problem (6). Unfortunately, it is found that the formulation in (6) is a highly nonlinear and nonconvex problem and hence difficult to solve. To make the problem more tractable, we next decouple it into three sub-problems and present an iterative algorithm to alternate between the sub-problems.

The first sub-problem is to find the optimal decoder matrices $\boldsymbol{W}_{1}$ and $\boldsymbol{W}_{2}$ given the precoding matrices at source and relay nodes, i.e., $\boldsymbol{A}_{1}, \boldsymbol{A}_{2}$ and $\boldsymbol{A}_{r}$. Since the power constraints in (1) and (2) are not related to $\boldsymbol{W}_{1}$ and $\boldsymbol{W}_{2}$, we can directly set the gradient of objective function in (6) to zero to get the optimal solutions. Namely,

$$
\partial J / \partial \boldsymbol{W}_{i}^{*}=0 \rightarrow \boldsymbol{W}_{i}^{\text {opt }}=\boldsymbol{A}_{\bar{i}}^{H} \boldsymbol{H}_{\bar{i}}^{H} \boldsymbol{A}_{r}^{H} \boldsymbol{G}_{i}^{H} \boldsymbol{R}_{W_{i}}^{-1},
$$

Second, we consider the optimization of the precoding matrix $\boldsymbol{A}_{r}$ at the relay node by assuming that $\boldsymbol{W}_{i}, \boldsymbol{A}_{i}(i=1,2)$

\footnotetext{
${ }^{1}$ Note that all the proofs in the paper are omitted due to space limitation.
} 
are fixed. From (6), the resultant optimization problem can be equivalently written as

$$
\begin{aligned}
& \min _{\boldsymbol{A}_{r}} J_{r_{1}}+J_{r_{2}}, \text { s.t. } \operatorname{Tr}\left\{\boldsymbol{A}_{r} \boldsymbol{R}_{x} \boldsymbol{A}_{r}^{H}\right\} \leq \tau_{r} \\
& J_{r_{i}}=\operatorname{Tr}\left\{\boldsymbol{G}_{i}^{H} \boldsymbol{W}_{i}^{H} \boldsymbol{W}_{i} \boldsymbol{G}_{i} \boldsymbol{A}_{r} \boldsymbol{R}_{x_{\bar{i}}} \boldsymbol{A}_{r}^{H}-\boldsymbol{H}_{\bar{i}} \boldsymbol{A}_{\bar{i}} \boldsymbol{W}_{i} \boldsymbol{G}_{i} \boldsymbol{A}_{r}\right. \\
& \left.-\boldsymbol{G}_{i}^{H} \boldsymbol{W}_{i}^{H} \boldsymbol{A}_{\bar{i}}^{H} \boldsymbol{H}_{\bar{i}}^{H} \boldsymbol{A}_{r}^{H}+\sigma_{i}^{2} \boldsymbol{W}_{i} \boldsymbol{W}_{i}^{H}+\boldsymbol{I}_{N}\right\}, i=1,2
\end{aligned}
$$

where $\boldsymbol{R}_{x_{i}}=\boldsymbol{H}_{i} \boldsymbol{A}_{i} \boldsymbol{A}_{i}^{H} \boldsymbol{H}_{i}^{H}+\sigma_{i}^{2} \boldsymbol{I}_{N_{r}}, i=1,2$ and $\boldsymbol{R}_{x}=$ $\boldsymbol{H}_{1} \boldsymbol{A}_{1} \boldsymbol{A}_{1}^{H} \boldsymbol{H}_{1}^{H}+\boldsymbol{H}_{2} \boldsymbol{A}_{2} \boldsymbol{A}_{2}^{H} \boldsymbol{H}_{2}^{H}+\sigma_{r}^{2} \boldsymbol{I}_{N_{r}}$. Since the transmit power $\tau_{i}$ at $S_{i}$ is constant with fixed $\boldsymbol{A}_{i}$, it is irrelevant here. To solve $\boldsymbol{A}_{r}$, we first give the following lemma.

Lemma 1: For fixed $\boldsymbol{W}_{1}, \boldsymbol{W}_{2}, \boldsymbol{A}_{1}$ and $\boldsymbol{A}_{2}$, the optimization problem (8) is convex with respect to the relay precoder $\boldsymbol{A}_{r}$.

Thus, we can readily design the optimal relay precoder by employing the KKT conditions. In specific, the Lagrangian function of (8) is given as

$$
\mathcal{L}=J_{r_{1}}+J_{r_{2}}+\lambda\left(\operatorname{Tr}\left\{\boldsymbol{A}_{r} \boldsymbol{R}_{x} \boldsymbol{A}_{r}^{H}\right\}-\tau_{r}\right),
$$

where $\lambda \geq 0$ is the Lagrange multiplier associated with the power constraint. Thus, the KKT conditions are shown to be

$$
\begin{gathered}
\frac{\partial \mathcal{L}}{\partial \boldsymbol{A}_{r}^{*}}=\boldsymbol{R}_{r_{1}} \boldsymbol{A}_{r} \boldsymbol{R}_{x_{2}}+\boldsymbol{R}_{r_{2}} \boldsymbol{A}_{r} \boldsymbol{R}_{x_{1}}-\boldsymbol{R}_{r}+\lambda \boldsymbol{A}_{r} \boldsymbol{R}_{x}=\mathbf{0}, \\
\lambda\left(\operatorname{Tr}\left\{\boldsymbol{A}_{r} \boldsymbol{R}_{x} \boldsymbol{A}_{r}^{H}\right\}-\tau_{r}\right)=0 \\
\operatorname{Tr}\left\{\boldsymbol{A}_{r} \boldsymbol{R}_{x} \boldsymbol{A}_{r}^{H}\right\} \leq \tau_{r},
\end{gathered}
$$

where $\boldsymbol{R}_{r}=\boldsymbol{G}_{1}^{H} \boldsymbol{W}_{1}^{H} \boldsymbol{A}_{2}^{H} \boldsymbol{H}_{2}^{H}+\boldsymbol{G}_{2}^{H} \boldsymbol{W}_{2}^{H} \boldsymbol{A}_{1}^{H} \boldsymbol{H}_{1}^{H}$ and $\boldsymbol{R}_{r_{i}}=$ $\boldsymbol{G}_{i}^{H} \boldsymbol{W}_{i}^{H} \boldsymbol{W}_{i} \boldsymbol{G}_{i}, i=1,2$. To satisfy the above KKT condition$\mathrm{s}$, two cases can be derived.

Case 1: $\lambda=0$. Substituting $\lambda=0$ into (9), we get

$$
\boldsymbol{A}_{r}^{\text {opt }}=\operatorname{mat}\left\{\left[\boldsymbol{R}_{x_{2}} \otimes \boldsymbol{R}_{r_{1}}+\boldsymbol{R}_{x_{1}} \otimes \boldsymbol{R}_{r_{2}}\right]^{-1} \operatorname{vec}\left(\boldsymbol{R}_{r}\right)\right\} .
$$

Note that this solution should satisfy the power constraint (11). Case 2: $\lambda>0$. From (9), we have

$$
\begin{aligned}
\boldsymbol{A}_{r}^{\text {opt }}= & \operatorname{mat}\left\{\left[\boldsymbol{R}_{x_{2}} \otimes \boldsymbol{R}_{r_{1}}+\boldsymbol{R}_{x_{1}} \otimes \boldsymbol{R}_{r_{2}}\right.\right. \\
& \left.\left.+\lambda \boldsymbol{R}_{x} \otimes \boldsymbol{I}_{N_{r}}\right]^{-1} \operatorname{vec}\left(\boldsymbol{R}_{r}\right)\right\} .
\end{aligned}
$$

In this case, $\lambda$ should be chosen such that $\operatorname{Tr}\left\{\boldsymbol{A}_{r} \boldsymbol{R}_{x} \boldsymbol{A}_{r}^{H}\right\}=$ $\tau_{r}$ in order to satisfy the condition (10).

To find $\lambda$, we have the following lemma.

Lemma 2: For the solution (13), the function $g=$ $\operatorname{Tr}\left\{\boldsymbol{A}_{r} \boldsymbol{R}_{x} \boldsymbol{A}_{r}^{H}\right\}$ is monotonicly decreasing with respect to $\lambda$ and the optimal $\lambda$ is bounded within $\left(0, \sqrt{\frac{\operatorname{Tr}\left\{\boldsymbol{R}_{r} \boldsymbol{R}_{x}^{-1} \boldsymbol{R}_{r}^{H}\right\}}{\tau_{r}}}\right)$.

With Lemma 2, we can then apply the bisection search method to find the optimal $\lambda$ efficiently.

The third sub-problem is to optimize the source precoder $\boldsymbol{A}_{1}$ and $\boldsymbol{A}_{2}$ for fixed $\boldsymbol{A}_{r}, \boldsymbol{W}_{1}$ and $\boldsymbol{W}_{2}$. This is formulated as:

$$
\begin{aligned}
\min _{\boldsymbol{A}_{1}, \boldsymbol{A}_{2}} & J_{s}=J_{s_{1}}+J_{s_{2}}, \\
\text { s.t. } & \operatorname{Tr}\left\{\boldsymbol{A}_{i} \boldsymbol{A}_{i}^{H}\right\} \leq \tau_{i} \\
J_{s_{i}} & =\operatorname{Tr}\left\{\boldsymbol{R}_{s_{i 1}} \boldsymbol{A}_{\bar{i}} \boldsymbol{A}_{\bar{i}}^{H}-2 \Re\left(\boldsymbol{R}_{s_{i 2}} \boldsymbol{A}_{\bar{i}}\right)+\boldsymbol{R}_{s_{i 3}}\right\}, i=1,2 \\
& \operatorname{Tr}\left\{\boldsymbol{R}_{p_{1}} \boldsymbol{A}_{1} \boldsymbol{A}_{1}^{H}+\boldsymbol{R}_{p_{2}} \boldsymbol{A}_{2} \boldsymbol{A}_{2}^{H}\right\} \leq \tau^{\prime}{ }_{r}
\end{aligned}
$$

where $\boldsymbol{R}_{s_{i 1}}=\boldsymbol{H}_{\bar{i}}^{H} \boldsymbol{A}_{r}^{H} \boldsymbol{G}_{i}^{H} \boldsymbol{W}_{i}^{H} \boldsymbol{W}_{i} \boldsymbol{G}_{i} \boldsymbol{A}_{r} \boldsymbol{H}_{\bar{i}}, \boldsymbol{R}_{s_{i 2}}=$ $\boldsymbol{W}_{i} \boldsymbol{G}_{i} \boldsymbol{A}_{r} \boldsymbol{H}_{\bar{i}}, \quad \boldsymbol{R}_{s_{i 3}}=\sigma_{r}^{2} \boldsymbol{W}_{i} \boldsymbol{G}_{i} \boldsymbol{A}_{r} \boldsymbol{A}_{r}^{H} \boldsymbol{G}_{i}^{H} \boldsymbol{W}_{i}^{H}+$ $\sigma_{i}^{2} \boldsymbol{W}_{i} \boldsymbol{W}_{i}^{H}+\boldsymbol{I}_{N}, \quad \boldsymbol{R}_{p_{i}}=\boldsymbol{H}_{i}^{H} \boldsymbol{A}_{r}^{H} \boldsymbol{A}_{r} \boldsymbol{H}_{i}, \quad i=1,2$, and $\tau^{\prime}{ }_{r}=\tau_{r}-\sigma_{r}^{2} \operatorname{Tr}\left\{\boldsymbol{A}_{r} \boldsymbol{A}_{r}^{H}\right\}$. It is noted that the change of precoder at two sources can influence the power constraint at the relay. Hence, the relay power constraint should be included in optimization (14). Similar with Lemma 1, we can also prove that the optimization (14) is convex. We further show that it can be transformed into the convex quadratically constrained quadratic program (QCQP) problem. Thus it can be efficiently solved by applying available software package.

In summary, we outline the iterative total MMSE Based precoding design algorithm as follows (Algorithm 1):

- Initialize $\boldsymbol{A}_{i}=\sqrt{\frac{\tau_{i}}{N}} \boldsymbol{I}_{N}(i=1,2), \boldsymbol{A}_{r}=\sqrt{\frac{\tau_{r}}{\operatorname{Tr}\left\{\boldsymbol{R}_{x}\right\}}} \boldsymbol{I}_{N_{r}}$

- Repeat

- Update the decoder matrices $\boldsymbol{W}_{1}$ and $\boldsymbol{W}_{2}$ using (7) for fixed $\boldsymbol{A}_{1}$, $\boldsymbol{A}_{2}$ and $\boldsymbol{A}_{r}$;

- Update the relay precoder matrices $\boldsymbol{A}_{r}$ using (12) or (13) for fixed $\boldsymbol{A}_{1}, \boldsymbol{A}_{2}, \boldsymbol{W}_{1}$ and $\boldsymbol{W}_{2}$;

- For fixed $\boldsymbol{A}_{r}, \boldsymbol{W}_{1}$ and $\boldsymbol{W}_{2}$, solve the convex QCQP problem to get the optimal $\boldsymbol{A}_{1}$ and $\boldsymbol{A}_{2}$;

- Until the sum MSE $J_{1}+J_{2}$ converges.

Since in Algorithm 1, the solution for each subproblem is optimal, we conclude that the total MSE value is decreased for each iteration. Meanwhile, the total MSE is lower bounded. Hence, Algorithm 1 is convergent.

\section{Design Method 2: Channel Parallelization BASED PRECODING DESIGN}

It has been proved in [8] that the optimal precoding structure in one-way relaying should parallelize the channels between the source and the relay, as well as between the relay and the destination. We are thus motivated to design $\mathbf{A}_{1}, \mathbf{A}_{2}$ and $\mathbf{A}_{r}$ so as to simultaneously parallelize the bidirectional links in the MIMO two-way relay system. In this section, we first propose a channel parallelization method suitable for two-way commuincations, then show that, based on this method, the precoder design is reduced to a power allocation problem. To make the channel parallelization feasible, we only consider the case $N_{r}=N$ in this section.

\section{A. Proposed Precoder Structure}

We first apply the generalized SVD (GSVD) technique to jointly decompose the channel matrix pair $\left(\boldsymbol{H}_{1}, \boldsymbol{H}_{2}\right)$ in the MAC phase. In specific, $\boldsymbol{H}_{i}$ can be expressed as $\boldsymbol{H}_{i}=$ $\boldsymbol{V}_{h} \boldsymbol{\Sigma}_{h i} \boldsymbol{U}_{h i}^{H}$ with $\boldsymbol{V}_{h}$ being non-singular $N \times N$ complex matrix, $\boldsymbol{U}_{h i}$ being $N \times N$ unitary matrices, $\boldsymbol{\Sigma}_{h i}$ being $N \times N$ positive diagonal matrices. We see that if the relay precoder $\boldsymbol{A}_{r}$ contains $\boldsymbol{V}_{h}^{-1}$ at the right side and the source precoder $\boldsymbol{A}_{i}$ has $\boldsymbol{U}_{h i}$ at the left side, then the channels in the MAC phase can be parallelized. For the BC phase, since the superimposed signals should be simultaneously transmitted to two destination nodes, we construct one virtual point-to-point MIMO channel as $\boldsymbol{G}=\left[\boldsymbol{G}_{1}^{T}, \boldsymbol{G}_{2}^{T}\right]^{T}$. By imposing SVD technique on $\boldsymbol{G}$, we have $\boldsymbol{G}=\boldsymbol{V}_{g} \boldsymbol{\Lambda}_{g} \boldsymbol{U}_{g}^{H}$ with $\boldsymbol{V}_{g}$ and $\boldsymbol{U}_{g}$ being $2 N \times 2 N$ and $N \times N$ unitary matrices, $\boldsymbol{\Lambda}_{g}=\left[\boldsymbol{\Sigma}_{g}^{T}, \mathbf{0}_{N \times N}^{T}\right]^{T}$ and $\boldsymbol{\Sigma}_{g}$ being an $N \times N$ positive diagonal matrix. If $\boldsymbol{A}_{r}$ 
contains $\boldsymbol{U}_{g}$ at its left side, the virtual point-to-point MIMO channel $G$ is parallelized in the BC phase. For $G_{1}$ and $\boldsymbol{G}_{2}$, we have $\boldsymbol{G}_{1}=\boldsymbol{V}_{g 1} \boldsymbol{\Sigma}_{g} \boldsymbol{U}_{g}^{H}, \boldsymbol{G}_{2}=\boldsymbol{V}_{g 2} \boldsymbol{\Sigma}_{g} \boldsymbol{U}_{g}^{H}$ with $\boldsymbol{V}_{g 1}=\boldsymbol{V}_{g}(1: N, 1: N)$ and $\boldsymbol{V}_{g 2}=\boldsymbol{V}_{g}(N+1: 2 N, 1: N)$ (MATLAB notation for sub-matrix is adopted here). It is noted that $\boldsymbol{V}_{g 1}$ and $\boldsymbol{V}_{g 2}$ lose the orthogonal property.

Based on the above findings, we propose the following structure for the three precoders as

$$
\boldsymbol{A}_{i}=\boldsymbol{U}_{h i} \boldsymbol{\Sigma}_{A_{i}} \boldsymbol{V}_{A_{i}}, i=1,2, \quad \boldsymbol{A}_{r}=\boldsymbol{U}_{g} \boldsymbol{\Sigma}_{A_{r}} \boldsymbol{V}_{h}^{-1},
$$

where $\boldsymbol{V}_{A_{i}}$ is an arbitrary unitary matrix, $\boldsymbol{\Sigma}_{A_{i}}$ and $\boldsymbol{\Sigma}_{A_{r}}$ are $N \times N$ real diagonal matrices to be optimized in the next subsection. As a result, the received signals $\boldsymbol{y}_{i}$ in (3) can be rewritten as

$$
\boldsymbol{y}_{i}=\boldsymbol{V}_{g i} \boldsymbol{\Sigma}_{g} \boldsymbol{\Sigma}_{A r} \boldsymbol{\Sigma}_{h \bar{i}} \boldsymbol{\Sigma}_{A \bar{i}} \tilde{\boldsymbol{s}}_{\bar{i}}+\boldsymbol{V}_{g i} \boldsymbol{\Sigma}_{g} \boldsymbol{\Sigma}_{A r} \tilde{\boldsymbol{n}}_{r}+\boldsymbol{n}_{i}
$$

where $\tilde{\boldsymbol{s}}_{i}=\boldsymbol{V}_{A_{i}} \boldsymbol{s}_{i}$ and $\tilde{\boldsymbol{n}}_{r}=\boldsymbol{V}_{h}^{-1} \boldsymbol{n}_{r}$. Since $\boldsymbol{V}_{A_{i}}$ is unitary, it does not affect the statistical property of $\boldsymbol{s}_{i}$.

\section{B. Joint Power Allocation}

By substituting (15) and $\boldsymbol{W}_{i}^{\text {opt }}$ in (7) into (4), we have

$$
\begin{aligned}
J_{i} & =\operatorname{Tr}\left[\boldsymbol{I}_{L}+\left(\boldsymbol{\Sigma}_{A \bar{i}} \boldsymbol{\Sigma}_{h \bar{i}} \boldsymbol{\Sigma}_{A r} \boldsymbol{\Sigma}_{g}\right)\right. \\
& \left.\left(\sigma_{i}^{2} \boldsymbol{B}_{g i}+\sigma_{r}^{2} \boldsymbol{\Sigma}_{g} \boldsymbol{\Sigma}_{A r} \boldsymbol{B}_{h} \boldsymbol{\Sigma}_{A r} \boldsymbol{\Sigma}_{g}\right)^{-1}\left(\boldsymbol{\Sigma}_{g} \boldsymbol{\Sigma}_{A r} \boldsymbol{\Sigma}_{h \bar{i}} \boldsymbol{\Sigma}_{A \bar{i}}\right)\right]^{-1},
\end{aligned}
$$

where $\boldsymbol{B}_{g i}=\left(\boldsymbol{V}_{g i}^{H} \boldsymbol{V}_{g i}\right)^{-1}$ and $\boldsymbol{B}_{h}=\left(\boldsymbol{V}_{h}^{H} \boldsymbol{V}_{h}\right)^{-1}$. It is found that, although $J_{1}$ and $J_{2}$ have been simplified, the MSE matrices are still non-diagonal. Solving the optimization problem directly is difficult. However, we can resort to a tractable upper bound on the MSE to simplify the problem.

Lemma 3: Let the diagonal matrices $\boldsymbol{\Lambda}_{B g i}$ and $\boldsymbol{\Lambda}_{B h}$ contain the diagonal entries of $\boldsymbol{B}_{g i}$ and $\boldsymbol{B}_{h}$. Then, $J_{i}$ can be upper bounded as

$$
\begin{aligned}
J_{i} \leq & \operatorname{Tr}\left[\boldsymbol{I}_{L}+\left(\boldsymbol{\Sigma}_{A \bar{i}} \boldsymbol{\Sigma}_{h \bar{i}} \boldsymbol{\Sigma}_{A r} \boldsymbol{\Sigma}_{g}\right)\right. \\
& \left.\left(\sigma_{i}^{2} \boldsymbol{\Lambda}_{B g i}+\sigma_{r}^{2} \boldsymbol{\Sigma}_{g} \boldsymbol{\Sigma}_{A r} \boldsymbol{\Lambda}_{B h} \boldsymbol{\Sigma}_{A r} \boldsymbol{\Sigma}_{g}\right)^{-1}\left(\boldsymbol{\Sigma}_{g} \boldsymbol{\Sigma}_{A r} \boldsymbol{\Sigma}_{h \bar{i}} \boldsymbol{\Sigma}_{A \bar{i}}\right)\right]^{-1},
\end{aligned}
$$

It can be seen that the MSE matrices within the upper bounds have a simple diagonal structure. Therefore, we can minimize the upper bounds to design the precoders. We further assume $\boldsymbol{P}_{k}=\boldsymbol{\Sigma}_{k}^{2}$ with $k \in\{A 1, A 2, A r, g, h 1, h 2\}$, the upper bound in Lemma 3 denoted as $J_{i}^{u}$ can be reformulated as

$$
J_{i}^{u}=\sum_{n=1}^{N}\left(1+\frac{p_{g}^{n} p_{A r}^{n} p_{h \bar{i}}^{n} p_{A \bar{i}}^{n}}{\sigma_{1}^{2} \lambda_{B g i}^{n}+\sigma_{r}^{2} \lambda_{B h}^{n} p_{g}^{n} p_{A r}^{n}}\right)^{-1}
$$

where $p_{k}^{n}$ 's are the diagonal entries of $\boldsymbol{P}_{k}$ and $\lambda_{k}^{n}$ 's with $k \in\{B g, B h 1, B h 2\}$ are the diagonal entries of $\boldsymbol{\Lambda}_{k}$. It is interesting to find that $J_{i}^{u}$ is the summation of the MSE of each sub-parallelized channel after zero forcing $\boldsymbol{y}_{i}$ by $\boldsymbol{V}_{g i}^{-1}$.
Finally, the precoder design can be simplified to the optimization problem as follows:

$$
\begin{gathered}
\min _{p_{A i}^{n}, p_{A r}^{n}, \forall n} J^{u}=J_{1}^{u}+J_{2}^{u}, \\
\text { s.t. } \sum_{n=1}^{N} p_{A i}^{n} \leq \tau_{i}, i=1,2 \\
\sum_{n=1}^{N} p_{A r}^{n}\left(p_{h 1}^{n} p_{A 1}^{n}+p_{h 2}^{n} p_{A 2}^{n}+\sigma_{r}^{2} \lambda_{B h}^{n}\right) \leq \tau_{r}, p_{A i}^{n} \geq 0 .
\end{gathered}
$$

Compared to $J_{i}$, the expression in (17) exhibits a simpler form and is more analytically tractable. However, it is still a nonconvex optimization problem. In the following, we apply the iterative approach to convert the original optimization problem into two convex sub-problems.

1) Sub-problem 1: For given $p_{A 1}^{n}$ and $p_{A 2}^{n}, \forall n$, we optimize the programming as follows to get the optimal $\boldsymbol{P}_{A r}$

$$
\begin{array}{cl} 
& \min _{p_{A r}^{n}, \forall n} J^{u p}=J_{1}+J_{2} \\
\text { s.t. } & \sum_{n=1}^{N} p_{A r}^{n}\left(p_{h 1}^{n} p_{A 1}^{n}+p_{h 2}^{n} p_{A 2}^{n}+\sigma_{r}^{2} \lambda_{B h}^{n}\right) \leq \tau_{r}, \mathrm{p}_{A r}^{n} \geq 0, \forall n
\end{array}
$$

By verifying $\frac{\partial^{2} J_{i}}{\partial p_{A r}^{n}{ }^{2}}>0$, we conclude that this suboptimization problem is convex. Based on the KKT conditions, we derive the water-filling solution

$$
p_{A r}^{n}=\max [0, \operatorname{Root}(f)], \quad \forall n
$$

where Root denotes the maximum real root of the equation $f$ and $f$ is denoted as

$$
\begin{aligned}
& \mu\left(p_{h 1}^{n} p_{A 1}^{n}+p_{h 2}^{n} p_{A 2}^{n}+\sigma_{r}^{2} \lambda_{B h}^{n}\right)=\left(\sigma_{1}^{2} \lambda_{B g 1}^{n} p_{h 2}^{n} p_{g}^{n} p_{A 2}^{n}\right) / \\
& {\left[\sigma_{1}^{2} \lambda_{B g 1}^{n}+p_{A r}^{n}\left(\sigma_{r}^{2} \lambda_{B h}^{n} p_{g}^{n}+p_{g}^{n} p_{h 2}^{n} p_{A 2}^{n}\right)\right]^{2}+\left(\sigma_{2}^{2} \lambda_{B g 2}^{n} p_{h 1}^{n} p_{g}^{n} p_{A 1}^{n}\right)} \\
& /\left[\sigma_{2}^{2} \lambda_{B g 2}^{n}+p_{A r}^{n}\left(\sigma_{r}^{2} \lambda_{B h}^{n} p_{g}^{n}+p_{g}^{n} p_{h 1}^{n} p_{A 1}^{n}\right)\right]^{2},
\end{aligned}
$$

the variable $\mu$ should be chosen to consume all the relay power.

2) Sub-problem 2: For given $p_{A r}^{n}, \forall n$, we obtain $p_{A 1}^{n}$ and $p_{A 2}^{n}$ by solving the optimization problem as follows:

$$
\begin{aligned}
& \min _{p_{A 1}^{n}, p_{A 2}^{n}, \forall n} J^{u}=J_{1}^{u}+J_{2}^{u}, \\
& \text { s.t. } \sum_{n=1}^{N} p_{A i}^{n} \leq \tau_{i}, i=1,2 \\
& \qquad \sum_{n=1}^{N} p_{A r}^{n}\left(p_{h 1}^{n} p_{A 1}^{n}+p_{h 2}^{n} p_{A 2}^{n}+\sigma_{r}^{2} \lambda_{B h}^{n}\right) \leq \tau_{r}, \quad p_{A i}^{n} \geq 0,
\end{aligned}
$$

This sub-problem is also convex as $\frac{\partial^{2} J^{u}}{\partial p_{A i}^{n}{ }^{2}}>0$. However, the closed-form solution to this problem is generally not available. Some standard numerical methods, such as interiorpoint method, can be used to get the optimum solutions.

From sub-problem 1 and sub-problem 2 it is seen that $\boldsymbol{P}_{A r}$, $\boldsymbol{P}_{A 1}$ and $\boldsymbol{P}_{A 2}$ are tightly coupled. Thus, we apply an iterative approach to find the final solutions. As verified by our simulation, the algorithm converges in only a few iterations. After obtaining $\Sigma_{A_{1}}, \Sigma_{A_{2}}$ and $\Sigma_{A_{r}}$ from the square root of $\boldsymbol{P}_{A_{1}}, \boldsymbol{P}_{A_{2}}$ and $\boldsymbol{P}_{A_{r}}$, we substitute them into (15) to get the precoders. 


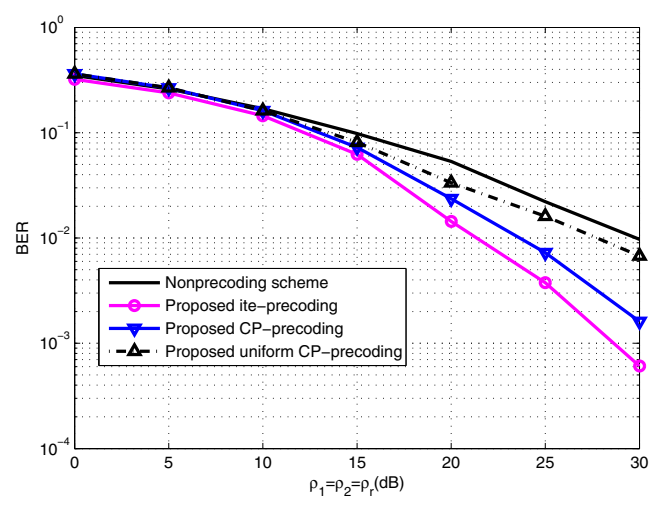

Fig. 2. The BER performance with $\rho_{1}=\rho_{2}=\rho_{r}$ at $N=N_{r}=2$.

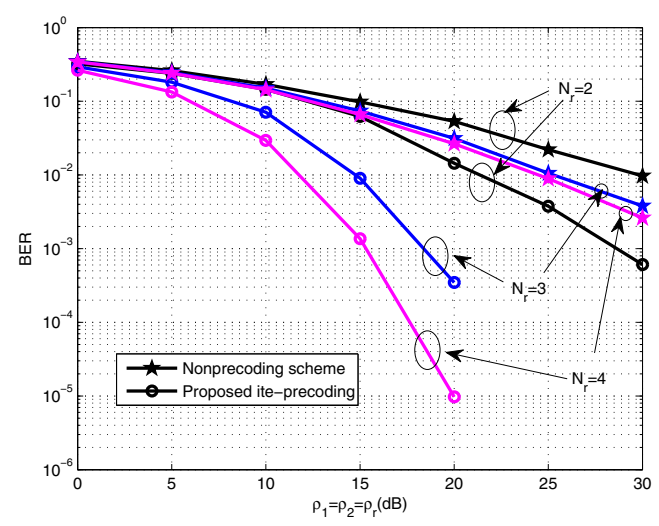

Fig. 3. The BER performance comparison at different relay antenna number.

\section{Simulation RESUlTS}

The noise power at two destinations are assumed equal to $\sigma^{2}$. The average SNR for the MAC and BC phases are defined as $\rho_{1}=\frac{\tau_{1}}{\sigma_{r}^{2}}, \rho_{2}=\frac{\tau_{2}}{\sigma_{r}^{2}}$ and $\rho_{r}=\frac{\tau_{r}}{\sigma^{2}}$, respectively.

In Fig. 2 , we show the bit-error-rate (BER) performance of the proposed two joint precoding methods, namely, the iterative method in Section III (ite-precoding) and the channelparallelization based precoding method in Section IV (CPprecoding) as the function of $\rho_{1}=\rho_{2}=\rho_{r}$ at $N=N_{r}=2$. For comparison, the $\mathrm{CP}$ precoding scheme with uniform power allocation (uniform CP-precoding) is also simulated. From the figure, we find that all the proposed precoding algorithms, including the Uniform-CP precoding, significantly increase the system BER performance. The iterative precoding algorithm exhibits the best performance among all the considered precoding methods. Furthermore, for the channel parallelization approach, optimizing the power allocation among each datastream can further enhance the system performance.

Fig. 3 illustrates the BER performance comparison at different $N_{r}$ when $N=2$. We find that increasing the relay antennas significantly enhances the BER performance thanks to the increased diversity gain. It is also seen that the proposed precoding scheme can get more performance gains compared to the nonprecoding scheme when the relay node is equipped more antennas.

Finally, the performance comparison between the proposed

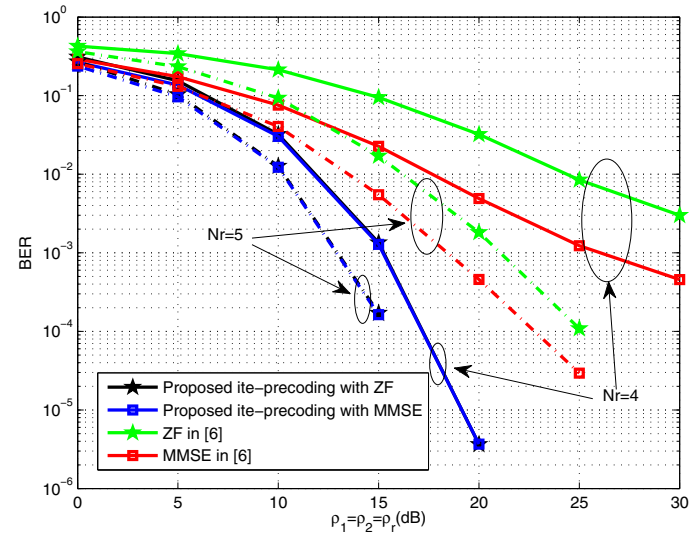

Fig. 4. The BER performance comparison with [6].

joint source/relay precoding algorithm and the relay precoding scheme in [6] at $N=2$ is shown in Fig. 4. Since the antenna configuration in [6] must satisfy $N_{r} \geq 2 N, N_{r}=4$ and 5 are chosen in the simulation. It is shown that, either applying MMSE or ZF receiver, the proposed joint precoding algorithm significantly outperforms the scheme in [6] where the precoding is applied at the relay only.

\section{CONCLUSION}

In this paper, we studied the joint precoding design for a two-way relay system based on the MMSE criterion. We first proposed an iterative method to obtain the local optimal solutions. Then, for the scenario in which all the nodes are equipped with the same number of antennas, we proposed a channel parallelization based precoding method to parallelize the channels in the MAC and BC phases. By doing so, the precoder design is converted into the simple power allocation problem. Simulation results showed that the performance is significantly increased by the proposed joint design algorithms.

\section{REFERENCES}

[1] B. Rankov and A. Wittneben, "Spectral efficient protocols for half-duplex fading relay channels," IEEE J. Sel. Areas Commun., vol. 25, no. 2, pp. 379-389, 2007.

[2] I. Hammerstrom, M. Kuhn, C. Esli, J. Zhao, A. Wittneben, and G. Bauch, "MIMO two-way relaying with transmit CSI at the relay," in Proc. IEEE 8th Workshop Signal Processing Advances in Wireless Communications SPAWC 2007, 2007, pp. 1-5.

[3] T. J. Oechtering, R. F. Wyrembelski, and H. Boche, "Multiantenna bidirectional broadcast channels - optimal transmit strategies," IEEE Trans. Signal Process., vol. 57, no. 5, pp. 1948-1958, 2009.

[4] R. Zhang, Y.-C. Liang, C. C. Chai, and S. Cui, "Optimal beamforming for two-way multi-antenna relay channel with analogue network coding," IEEE J. Sel. Areas Commun., vol. 27, no. 5, pp. 699-712, 2009.

[5] K.-J. Lee, K. W. Lee, H. Sung, and I. Lee, "Sum-rate maximization for two-way MIMO amplify-and-forward relaying systems," in Proc. IEEE 69th Vehicular Technology Conf. VTC Spring 2009, 2009, pp. 1-5.

[6] T. Unger and A. Klein, "Duplex schemes in multiple antenna two-hop relaying," EURASIP Journal on Advances in Signal Processing, 2008, DOI 101.1155/2008/128592.

[7] C. Li, X. Wang, L. Yang, and W.-P. Zhu, "A joint source and relay power allocation scheme for a class of MIMO relay systems," IEEE Trans. Signal Process., vol. 57, no. 12, pp. 4852-4860, 2009.

[8] R. Mo and Y. Chew, "MMSE-based joint source and relay precoding design for amplify-and-forward MIMO relay networks," IEEE Trans. Wireless Commun., vol. 8, no. 9, pp. 4668-4676, 2009. 